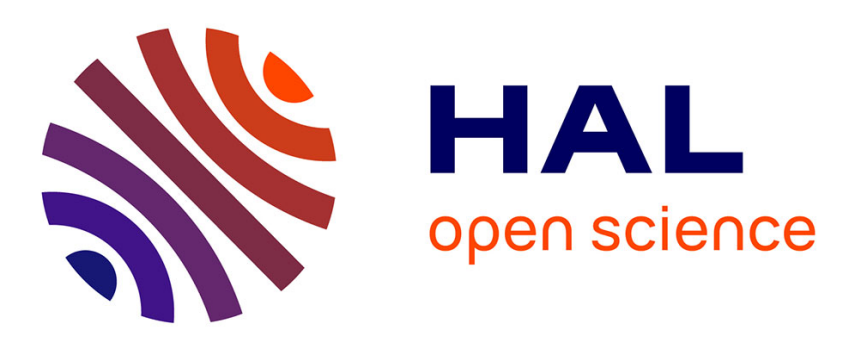

\title{
Exit to the past and voice for the future. Sciences de gestion, sciences fondamentales de l'action collective.
} Armand Hatchuel

\section{To cite this version:}

Armand Hatchuel. Exit to the past and voice for the future. Sciences de gestion, sciences fondamentales de l'action collective.. Revue Française de Gestion, 2019, 45 (285), pp.43-57. hal-02506096

\section{HAL Id: hal-02506096 \\ https: / hal-mines-paristech.archives-ouvertes.fr/hal-02506096}

Submitted on 12 Mar 2020

HAL is a multi-disciplinary open access archive for the deposit and dissemination of scientific research documents, whether they are published or not. The documents may come from teaching and research institutions in France or abroad, or from public or private research centers.
L'archive ouverte pluridisciplinaire HAL, est destinée au dépôt et à la diffusion de documents scientifiques de niveau recherche, publiés ou non, émanant des établissements d'enseignement et de recherche français ou étrangers, des laboratoires publics ou privés. 


\title{
Exit to the past and voice for the future. ${ }^{1}$
}

Sciences de gestion, sciences fondamentales de l'action collective.

Armand Hatchuel

MinesParisTech/PSL Université

CGS, I3, UMR 9217.

Cet article a été pubié dans Revue Française de Gestion vol. 45, n²85, nov-dec 2019 p.43-57

\section{Résumé}

Cet article résume les résultats d'une refondation des sciences de gestion. II repart de la "bene gesta " romaine qui reliait l'action rationnelle et la responsabilité citoyenne. Impensable dans la modernité " économiciste ", elle revient avec la théorie administrative de Fayol et de l'entreprise moderne. Pourtant, la théorie du management oublie fayol et se réduit à la quête de performances dans l'entreprise actionnariale. Aujourd'hui, relier rationalité et responsabilité fonde une science de l'action collective qui a contribué à la théorie de la conception et à la redéfinition de « l’entreprise » dans la Loi Française.

\section{Exit through the past and voice for the future.}

The conditions of a basic science of collective action.

\begin{abstract}
This article overviews research that builds new foundations for Management science. It begins with the rediscovery of the Roman " bene gesta " which coupled rationality and citizen responsibility. This linkage was unthinkable to the liberal modernity and its "economicism». It returned in Henri fayol's administrative theory where the creative rationality of science required solidarity in the unknown. The modern corporation came from it. Fayol forgotten, Management science was reduced to a quest of performance under the dogma of shareholder primacy. Now, the non-separability of rationality and responsibility paves the way to a basic science of collective action and creation. In addition to several theoretical and historical findings, like design theory, this research contributed to the new corporate law in France.
\end{abstract}

\section{Introduction : le chemin parcouru depuis les « nouvelles fondations »}

Cet article introduit quelques résultats, parfois surprenants, du programme de refondation des sciences de gestion lancé en 2001 (David et al. 2001). Dès ses débuts, ce programme exigeait un réexamen des sources historiques, de la terminologie et des questions originelles du champ. A terme, étaient visés la reconstitution d'une mémoire scientifique et un réarmement théorique de la recherche. Si l'on en juge par le chemin parcouru, cet exit to the past s'est révélé d'une réelle fécondité. Il a conduit, notamment aux quelques percées suivantes :

\footnotetext{
${ }^{1}$ Je tiens à remercier Jean-Philippe Denis, rédacteur en chef, ainsi que Alain-Charles Martinet et Pierre-Michel Menger, pour leur invitation. Par ailleurs, ce texte a bénéficié des suggestions de Jean-Philippe Denis, Blanche Segrestin, Kevin Levillain, Pascal Le Masson et Benoit Weil.
} 
- Une archéologie inédite de la " gestion » : elle a confirmé la nécessité de remonter aux sources antiques, donc bien en-deçà de la modernité libérale et du management américain de l'aprèsguerre (Hatchuel et Weil 2018, Crété 2018);

- Un modèle original de la rationalité créatrice (ou théorie C-K de la conception) ${ }^{2}$ (Hatchuel 2001b, Hatchuel et Weil 2014, Le Masson et al. 2013) : Cette rationalité conceptive était impensable dans le cadre de la logique décisionnelle classique. Elle éclaire, les conditions cognitives d'une action novatrice (technique, entrepreneuriale ou sociale) ainsi que les solidarités requises pour affronter collectivement un futur à la fois inconnu et désirable (Hatchuel et al. 2017) ${ }^{3}$.

- Une théorie axiomatique de l'action collective, et plus particulièrement de la création collective, qui repose sur le principe de non-séparabilité entre rationalité et responsabilité (Hatchuel 2005)

- Une nouvelle définition de "l'entreprise»" et de sa mission, adaptée aux défis de la civilisation contemporaine (Segrestin et Hatchuel 2011, 2012)

On ne peut examiner ici tous ces travaux et cet article ne présente que quelques avancées historiques et théoriques qui illustrent ce parcours. Ces résultats peuvent nourrir une discussion transdisciplinaire et ont une pertinence directe pour les problèmes contemporains. Au préalable, nous donnons ci-dessous un bref résumé des points développés dans ce texte.

1. En 1949 paraissait la seconde traduction anglaise du traité de Henri Fayol, mais le terme " management " y est substitué à " administration " dans le titre du livre. Lyndall Urwick, invité à écrire la préface, dénonce une trahison de la théorie Fayolienne, au nom d'un dogme de la modernité libérale anglo-saxonne (que nous appellerons " économicisme ».). L'histoire a donné raison à Urwick: ce détournement a masqué I'histoire des notions "d'administration " et de "gestion ", privant la recherche d'un héritage théorique sans lequel elle ne pouvait se consolider.

2. On sait, depuis peu, que gerere (notre "gérer ") a occupé une place intellectuelle et politique majeure dans la Rome classique. C'est là que s'invente une théorie de la bene gesta ("bonne gestion ") qui s'opposait aux pratiques anciennes de gouvernement de la cité. Son originalité résidait dans la fusion dans un même concept, de la rationalité de l'action et de la responsabilité citoyenne. Cependant, ce fonds théorique sera perdu avec ce que nous appelons l' « économicisme » de la fin du 18è siècle qui dissocie efficacité et responsabilité.

3. Un siècle plus tard, l'irruption de la science et de la recherche technique pose une énigme inédite à la modernité. II faut domestiquer une rationalité conceptrice, créatrice de progrès et de dangers... imprédictibles! Le futur devient en grande partie inconnu, et pour protéger chacun des risques à venir, il faut repenser la solidarité de l'action collective qu'elle soit privée ou publique. Emerge alors le projet Fayolien d'une "science administrative » renouvelée et unifiée. II recoupe les percées de Taylor, Follett et Barnard. "L'entreprise moderne", le contrat de travail et l'Etat protecteur naitront de ce faisceau d'inventions et des luttes sociales du 20 è siècle.

4. Mais comme l'avait prévu Urwick, le " management " américain de l'après-guerre se construit sur l'oubli de cet héritage (O'connor 2011). Sans unité théorique, et réduit à la recherche des facteurs de la performance, il ne pourra pas résister à la financiarisation des années 80 et aux codes de « bonne gouvernance » qui imposent une logique actionnariale.

\footnotetext{
${ }^{2}$ Le formalisme de cette rationalité créatrice découle de la théorie unifiée de la conception dite aussi Théorie C-K ( Concept-Knowledge theory, Hatchuel et Weil 2007)

${ }^{3}$ La rationalité créatrice (ou raison conceptive) échappe aux oppositions classiques entre rationalité et imagination, rationalité et désir, rationalité et émotions. Elle organise au contraire une dialectique constructive et générative entre ces différents termes (Hatchuel et Weil 2014)

${ }^{4}$ Et de la société commerciale qui lui sert de personnalité juridique.
} 
Aujourd'hui, la reconquête de cette mémoire, ainsi que de nombreux travaux, débouchent sur une théorie axiomatique de l'action collective d'une cohérence et d'une universalité inattendues. Au cœur de cette théorie, il y a l'essence de la bene gesta: le principe de non-séparabilité entre rationalité et responsabilité. Ce principe restaure notre capacité collective à inventer et à réaliser des futurs désirables. Il a aussi inspiré le projet de refondation de l'entreprise (Segrestin et Hatchuel 2012) qui a contribué à la nouvelle loi française sur l'entreprise responsable, sa raison d'être et sa mission.

\section{Le grand détournement : quand « management » fait oublier « administration »}

En 1949, l'éditeur anglais Sir Isaac Pitman \& sons confie à Constance Storrs ${ }^{5}$ une nouvelle traduction du traité d'Henri Fayol, Administration industrielle et générale publié en 1916. La première traduction due à J.A Coubrough avait été éditée à Genève en $1929^{6}$. Or, entre la traduction de Coubrough et celle de Storrs, il y a une différence immédiatement visible. Coubrough intitulait sa traduction "General and industrial Administration", mais vingt ans plus tard, Storrs abandonne "administration" et le traité devient "General and industrial Management ». Mais, ironie de I'histoire, la préface de l'ouvrage est confiée à, Lyndall Urwick, introducteur des travaux de Fayol dans le monde anglo-saxon, qui est en désaccord total avec le nouveau titre ! Et il va le faire savoir dans sa préface par une dénonciation peu commune: "Personnally, and taking the long view, i feel that it is a pity that Mrs Storrs and Mr. Pitman have decided to translate Fayol's word " administration » by " management ". In the original english translation his title was translated directly « administration».

Ensuite, Urwick explique longuement qu'il s'agit d'une erreur scientifique qui aura des conséquences sociales et politiques importantes. Car la nouvelle traduction s'aligne sur la tradition anglo-saxonne qui distingue clairement entre la notion de " management " pour les entreprises et celle de " public administration » réservée aux activités de l'Etat. Et cet alignement a un prix inacceptable : I'abandon d'un axiome central de la pensée fayolienne, l'unité de la « science de l'administration ». Car celle-ci doit s'appliquer aussi bien à l'entreprise privée qu'à l'action publique : "lt was of the essence of Fayol's teaching that this distinction is false and misleading ". Et Fayol tenait particulièrement à ce principe : " as has been shown he (Fayol) devoted much of his effort in the concluding years of his life to demonstrating this unity of administrative theory".

\section{L'alignement sur la tradition anglo-saxonne}

Urwick va droit au but : l'unité de la science administrative s'oppose à des principes idéologiques de la culture anglo-saxonne dans laquelle "the false antithesis between "business management » and public administration " is far more widespread and cherished more tenaciously by members of both callings ». L'éditeur et la traductrice ont donc choisi d'imposer cette tradition même si l'édifice théorique de Fayol est ainsi mis à bas, et alors même, martèle Urwick, que les spécialistes américains partagent la position de Fayol: "the attempt to subdivide the study of management or administration in accordance with the purpose of particular forms of undertaking seems to many authorities, as it seemed to Fayol himself, equally misdirected ".

Fayol s'opposait au dogme qui assigne à l'entreprise privée un objectif exclusif de profit et qui demande à l'acteur public de s'en tenir à rendre un service au citoyen. Car ce dogme est scientifiquement intenable. II réduit la science administrative à une recherche autiste de l'efficacité et I'on compterait alors autant de théories administratives que de critères d'efficacité ! En affirmant

\footnotetext{
${ }^{5}$ Constance Storrs $(1911,1990)$ : jeune enseignante en gestion des entreprises en 1949.

${ }^{6}$ Disparu en 1925, Fayol n'a connu aucune de ces traductions
} 
l'unité de la théorie administrative, Fayol fait de la définition de l'efficacité une conséquence de la théorie administrative et non l'inverse. La tache de cette dernière étant de chercher des invariants d'un ordre supérieur et indépendants des objectifs d'efficacité.

Urwick dénonçait un hold up langagier doublé d'un détournement théorique. Sa protestation se révéla prémonitoire. En intitulant "management » le traité de Fayol, on accréditait l'idée que son projet était une science de la performance des entreprises, science assujettie, par les dogmes libéraux, à des critères d'efficacité (profit) dont la définition était hors du champ de cette science. C'était un clair déni du pari théorique le plus audacieux et le plus actuel de Fayol : reconnaître que le choix des objectifs (purpose) de l'action collective, qu'il s'agisse des entreprises ou de l'Etat, n'est pas exogène à la science administrative mais en découle ${ }^{7}$. Car celle-ci est à la fois une science des moyens et une science des fins ! c'est-à-dire, une science qui étudie la bonne gestion de l'élaboration collective des fins. Ainsi, Fayol conditionnait la construction de son "programme d'action " à la recherche de "I'union du corps social » (Hatchuel et Segrestin 2019).

\section{Une longue amnésie conceptuelle}

Ce détournement allait favoriser une longue amnésie. Urwick rappelle que la notion $d^{\prime}$ '« administration » peut se réclamer d'un long héritage, alors que l'étymologie de " management » est récente et imprécise. Mais cette absence de passé avait un avantage. "Management " pouvait incarner un corpus récent, de source anglo-saxonne, alors que la science " administrative " évoquait un héritage latin. Mais Urwick refuse ce jeu des apparences et il assène : " they gave France a unified management body more than twenty years before the same ideal began to be realized in Great Britain "! Après 1949, Fayol fut reconnu comme un pionnier du "modern management ». Mais il s'agissait désormais d'une discipline nouvelle que l'on associait à la surprenante puissance industrielle des USA. En revanche, l'enseignement du Fayolisme fut vite réduit à quelques formules sans épaisseur ${ }^{8}$.

Quel était cet héritage ancien que la notion de "management " avait masqué ? L'ancienneté et l'universalité de la notion d'administration sont connues. Mais on ignorait, qu'à Rome, cette notion avait été associée à une théorie de la " bene gesta » (" bonne gestion ») dont l'influence durera plus de trois siècles.

\section{Bene gesta, l'invention romaine d'une théorie de de l'action collective}

La redécouverte récente de la bene gesta romaine par Moira Crété (Crété 2018) est une retombée inattendue du programme de refondation des sciences de gestion (Hatchuel et Weil 2018). La surprise, ce fut la révélation d'une notion de "gestion " dont la profondeur philosophique et politique avait été perdues ${ }^{9}$. Moira Crété montre qu'au cours de l'histoire romaine, gerere (gero, gestum,) suit une ascension qui va du sens commun " porter une charge, s'occuper de, se mettre au service d'autrui... " à la désignation d'un modèle normatif pour la conduite de l'action collective publique et privée.

L'élaboration philosophique de gerere et de la "bene gesta " a été principalement l'œuvre de Cicéron (106-43 av. ec.). Elle naît avec la crise de la république romaine (1è siècle av. ec.) dont le

\footnotetext{
${ }^{7}$ On sait que Fayol n’a pas manqué de le prouver dans sa propre pratique en imposant à ses actionnaires sa définition du " profit raisonnable ". Il le montre aussi en assignant à l'administration industrielle un objectif général de " perfectionnement » qui ne découle pas de la recherche du profit, mais de la prévoyance responsable.

${ }^{8}$ Ce n'est que depuis les années 90, et encore très récemment, à l'occasion du centenaire du Traité, que de nouvelles lectures explorent l'originalité et l'actualité de Fayol (Bertilorenzi et al. 2019, Hatchuel et Segrestin 2019).

${ }^{9}$ La notion est restée dans toutes les langues latines mais a quasiment disparu du lexique anglais.
} 
point de bifurcation fut l'assassinat de César et l'imposition de l'empire par Octave (Auguste). Avocat célèbre, homme politique et penseur, Cicéron cherche une issue à la crise politique en concevant, non un nouveau régime, mais une action collective réfléchie et responsable qui ne soit ni le pouvoir de l'élite, ni un populisme qui trahirait les valeurs de la cité. Avant Cicéron, gerere avait déjà un sens complexe en s'appliquant surtout à la guerre (de bellum gerere). Gerere ne se résume pas à l'obtention de la victoire à tout prix. Car même en cas de succès, une conduite irresponsable de la guerre, pour sa propre armée ou pour l'ennemi, serait porteuse de conséquences néfastes. Cet exemple illustre la matrice conceptuelle de Gerere que Cicéron généralise à l'ensemble des actions collectives. Pour lui, Gerere désigne : d'une part, l'action réfléchie, rationnelle, éclairée, efficace, appropriée à son contexte; et d'autre part, l'action bienveillante, intègre, respectueuse de la loi, et tout autant des vertus romaines.

\section{L'administration des relations citoyennes}

La " bonne gestion » romaine englobait dans un même concept une bonne administration des choses et une bonne administration des...relations citoyennes. Et il ne s'agit pas ici de l'administration des hommes ${ }^{10}$ ! Car cette expression occulte les responsabilités et les valeurs citoyennes qu'elle doit pourtant servir et défendre. La bene gesta se définit donc, et indissociablement, comme idéal d'efficacité et idéal de responsabilité. Car la responsabilité est précisément ce qui organise les relations citoyennes dans la cité. L'originalité théorique de la bene gesta réside dans le tressage continu de ces deux régimes de l'action collective en un unique concept. Elle décrit l'acte de gestion (ou d'administration) non comme un pouvoir "sur ", mais comme un pouvoir " pour » et comme un pouvoir "avec ». Chez Cicéron, elle convoque les valeurs aristocratiques de la citoyenneté romaine et étend celles-ci à l'exercice de toute responsabilité. Avec la bene gesta, les rapports entre gouvernants et gouvernés ne se décrivent plus comme l'exercice d'une puissance (cratos, Крó́тоऽ) mais comme des relations de réciprocité citoyenne entre hommes libres où l'autorité se construit et s'évalue par l'action au service des citoyens et par l'assentiment qu'elle reçoit en retour de ces derniers.

La théorie de la bonne gestion cicéronnienne aurait pu rester lettre morte. Elle reçut une légitimité prestigieuse de la part d'Auguste, le premier empereur. Elève de Cicéron, il s'en réclama publiquement dans son célèbre testament qui s'ouvre par l'expression:Res gestae divi Augusti...Cette invention théorique connut ensuite une très large diffusion. Durant les trois siècles qui suivirent le règne d'Auguste, les principes de la bene gesta constituèrent, dans tout l'empire, la norme de toute fonction dont on a reçu mandat (Crété 2018). Le déploiement de l'Empire romain n'aurait pas été possible sans de nouveaux acteurs (curateurs, administrateurs, sociétés, édiles...) qui assurèrent son expansion politique, technique et géographique. La "bene gesta " leur donna un cadre d'action raisonné et légitime.

\section{L'oubli de la bene gesta et la formation de l'économicisme}

Des travaux sont en cours pour suivre le destin de la bene gesta après la chute de l'empire romain $\mathrm{d}$ 'occident. Mais la redécouverte de cette invention suffit pour ouvrir une critique nouvelle de la modernité libérale. Car, elle invite à rejeter l'idée moderne qu'une action peut être jugée efficace ou rationnelle indépendamment de toute référence à un jugement de responsabilité, c'est-à-dire à une relation normée ou légitime vis-à-vis d'un autrui.

\footnotetext{
${ }^{10}$ On sait le succès chez les penseurs classiques du 19è siècle de la dualité : administration des choses et administration des hommes.
} 
Ainsi la séparation conceptuelle de la rationalité (ou de l'efficacité) et de la responsabilité a installé la modernité comme une antithèse de la bene gesta : celle-ci devenait impensable et son oubli favorisa un profond schisme conceptuel dans la compréhension de l'action collective. D'une part, la pensée politique, le droit puis, dans une large mesure, la sociologie se construisirent comme des théories de la responsabilité ou des relations sociales; d'autre part, l'Economie et certaines théories de la technique se définirent comme des théories de l'efficacité (ou de l'action rationnelle). Cette dissociation conduit tout naturellement à penser que l'action privée relève d'une gestion guidée par l'efficacité individuelle (ou le profit qui en est une traduction), alors que l'action publique relève avant tout de l'exercice d'une responsabilité vis-à-vis des gouvernés.

Nous appelons économicisme ${ }^{11}$ le principe de cette séparation, principe contre lequel protestait Urwick, au nom de Fayol. Précisons que l'étude du phénomène économique ne suppose pas l'économicisme. Comme pour tout "réel ", de multiples cadres théoriques sont possibles et nécessaires. L'économicisme est, en revanche, indispensable pour fonder certains dogmes de la modernité libérale et pour constituer le savoir économique (et non le phénomène) en discipline autonome. En effet, un savoir économique qui se veut science séparée et séparable exige un principe universel d'efficacité (le profit, l'utilité, le rapport cout/bénéfice, la concurrence parfaite...) indépendant de tout principe de responsabilité donc de toute théorie des relations citoyennes ${ }^{12}$. Si l'on suppose que chaque individu ne recherche que son propre intérêt, on postule simultanément que cet intérêt peut être défini sans responsabilité citoyenne vis-à-vis d'un autrui. Ainsi, pour un marchand toute bene gesta semble sans signification. II n'a pas à être bienveillant vis-à-vis de ses clients ; rien ne l'oblige à les traiter avec équité ! La relation entre un marchand et ses clients peut s'accommoder de toutes les démagogies et les simulacres possibles tant qu'ils ne sont pas interdits par la loi.

En réalité, un tel économicisme n'a jamais été tenable. Et, il a fallu les normes de gestion, imposées par la puissance publique, comme l'interdiction de la contrefaçon ou de la publicité mensongère, pour reconstituer une bene gesta marchande. D'où le paradoxe d'un économicisme marchand qui ignore les principes d'une bene gesta, mais qui ne survit, en réalité, que parce que la Loi ou l'intelligence du commerce ${ }^{13}$ impose les nécessités de l'action collective. Ainsi s'éclaire un autre trait surprenant de la doctrine fayolienne : l'absence de toute référence au savoir économique de son temps (Hatchuel et Segrestin 2019). La théorie administrative fayolienne se voulant universelle, il fallait éviter tout économicisme.

Depuis le début du 19è siècle, l'économicisme domine dans le langage des Etats, des experts et des médias. L'idée d'une science universelle de l'administration ne pouvait donc apparaître que comme une hérésie. Entre l'administration publique et la vie des affaires, une différence de nature devait être affirmée. Le monde des affaires y voyait la condition de la liberté d'entreprise et l'Etat moderne

\footnotetext{
${ }^{11}$ On remarquera que l'économicisme ne se confond pas avec un utilitarisme ou avec la notion d'homo economicus. L'économicisme nait de la séparation conceptuelle entre efficacité et responsabilité, quelquesoit la définition retenue pour l'efficacité. On peut donc concevoir une efficacité altruiste et qui reste pourtant un économicisme ou encore un économicisme non utilitariste, si on peut la définir sans référence à une théorie des relations citoyennes.

${ }^{12}$ On sait que le savoir économique, s'est longtemps intitulé " économie politique ». Cette dénomination montrait une prise de distance vis-à-vis de l'économicisme. Son abandon peut être considéré comme un marqueur d'une autonomisation du savoir économique qui conduit à l'économicisme. Il s'agit là d'une question d'histoire du savoir économique qui ne peut être traitée ici. En revanche, l'économicisme est une doctrine dont on peut attester l'influence dans la culture générale.

${ }^{13}$ Dans son « Traité du parfait négociant » (1675), Jacques Savary s'efforce de construire cette « intelligence du commerce ». Nous sommes un siècle avant la naissance du mouvement « libéral » et on a pu montrer que Savary pense l'activité marchande dans un cadre théorique différent (Cf. Colloque de Cerisy, l'activité marchande sans le marché).
} 
pouvait se prévaloir d'exprimer "l'intérêt général ». Mais il ne faudra pas attendre longtemps pour que le besoin d'une nouvelle théorie de la bonne gestion se fasse douloureusement sentir, autant pour l'Etat que pour les acteurs privés!

\section{La Science, l'inconnu et l'entreprise moderne : la renaissance d'une science de "la bonne gestion »}

L'irruption de la science et de la recherche technique dans tout le tissu des activités humaines a bouleversé les repères de l'action et notamment la frontière public-privé ${ }^{14}$. Elle impose, dès le milieu du 19è siècle, la recherche de nouvelles formes d'autorité et de nouveaux principes d'action collective. Le peuple de machines qui s'implante dans les ateliers, les bureaux, les ministères requiert des autorités, des principes d'efficacité, des modes d'obéissance au travail et d'assentiment différents de ce que l'on connaissait auparavant. Machinisme, technicisation, complexification des rapports de travail et des rapports marchands hantent les pensées politico-sociales du 19è et du 20è siècle. Ils nourrissent crises, luttes politiques et contre-cultures. Cette histoire est bien connue. Avec le recul du temps, on peut remarquer que les tentatives d'énonciation d'une nouvelle bene gesta sont venues de nouveaux acteurs (ingénieurs, savants, mouvements ouvriers.) et de nouvelles percées théoriques (marxisme, Economie sociale, coopératisme, Fayolisme, Taylorisme...) toutes indissociables de l'esprit scientifique. Sur une période courte de l'histoire (1880-1920), et au milieu des guerres meurtrières et des révolutions politiques, on voit émerger un "droit du travail ", une figure du "chef d'entreprise ", un monde "d'entreprises "; sans oublier un nouvel appareil d'Etat, ce dernier étant aussi sommé de repenser ses principes d'action. Partout s'inventent les agir collectifs qui ont fait le second 20 è siècle.

Ce détour historique et théorique réinscrit les pensées de Taylor, de Fayol et de Follett dans l'histoire longue de la civilisation. Chacun d'eux tentait de rebâtir une bene gesta contre les doctrines de la modernité libérale. L'activité industrielle ne fut pas la seule à devoir repenser ses principes d'efficacité et de responsabilité. Mary Parker Follett repense la gestion des organisations caritatives et humanitaires qui se multiplient aux USA avec les vagues d'émigration (O'connor 2011). Le monde militaire affrontait aussi une crise technique et une crise du commandement (Saint-Fuscien 2011). La mécanisation des armes décuplait la puissance de feu et modifiait considérablement la notion de bataille et son déroulement. La discipline et l'obéissance n'étaient plus garantes ni de l'efficacité au combat, ni d'un comportement responsable face à l'ennemi. En 1891, le futur Maréchal Lyautey, publie, "le rôle social de l'officier $~_{15}^{15}$ où il retrouve des accents de la bene gesta cicéronienne. Il veut repenser l'action et la responsabilité des officiers, car l'obéissance doit venir de la sollicitude des chefs et de l'éducation morale des soldats et celle-ci est le meilleur gage de leur engagement et de leur efficacité au combat.

\section{Un nouveau futur et les deux sens de la prévoyance}

Fayol était le mieux placé pour remarquer que la science brise fondamentalement les représentations classiques du futur. Elle met à mal la rationalité et la responsabilité des nouveaux "chefs d'entreprise ", tout autant que celles des gouvernements des Etats. D'une part, le futur devient inconnu ; d'autre part, toute autorité, privée ou publique, doit envisager une activité de recherche

\footnotetext{
${ }^{14}$ On sait aujourd'hui que cette irruption marque l'entrée dans l'anthropocène, où l'action collective humaine change l'histoire de la Terre.

${ }^{15}$ Revue des Deux Mondes, 3e période, tome 104, 1891 (p. 443-459).
} 
pour tenter de faire advenir ce qui est encore inconnu mais néanmoins désirable! ${ }^{16}$. La conception économiciste de l'entrepreneur (et de l'Etat) se trouve alors devant une impasse bien décrite par Frank Knight (Hatchuel et Segrestin 2019). Car l'investissement dans la recherche scientifique est un investissement dans l'inconnu, dont l'efficacité (rapport coût-bénéfice) est incalculable ou indécidable! Comment un chef d'entreprise pourrait-il alors justifier l'efficacité d'un tel investissement? La réponse de Fayol déjoue l'énigme : il serait simplement irresponsable pour un chef d'entreprise (comme pour un Etat) de ne pas « soutenir les savants " !

De façon violente, la question de la bene gesta revenait avec la modernité scientifique et rappelait l'inséparabilité de la rationalité et de la responsabilité. Car face à l'inconnu, à l'attente du surgissement, à la puissance créatrice, c'est la responsabilité qui s'impose comme principe de rationalité et qui pallie au calcul impossible ou désespérément myope. Fayol y trouve la première composante de sa théorie administrative: la prévoyance, qui acquiert avec la crise écologique contemporaine une pertinence encore plus grande.

On aurait dû s'étonner qu'un industriel mette en avant une notion qui désigne les systèmes d'entraide et d'assistance mutuelle! Certes, Fayol recommande de prévoir autant que possible le futur mais il reconnaît aussitôt l'imprédictibilité de l'avenir. D'où la double signification de sa prévoyance : face à l'inconnu menaçant, l'effort d'anticipation conduit, non à la prévision illusoire, mais à renforcer la solidarité et le soutien mutuel! Ainsi, dans la bene gesta moderne, la prévoyance, comprise comme l'anticipation efficace, et la prévoyance, vue comme l'organisation responsable de la solidarité, se rejoignent dans le même concept. En montrant qu'un terme aussi éloigné du monde des affaires est essentiel pour un chef d'entreprise, Fayol écarte toute confusion entre sa science administrative et une optimisation productive ou marchande. Il établit une proposition suffisamment universelle pour décrire les métamorphoses de la bonne gestion au cours de l'histoire et pour stimuler, si nécessaire, l'invention de formes nouvelles. C'est ainsi que face à l'inconnu inquiétant inhérent à la rationalité créative de la recherche, Fayol prescrit "l'union du corps social » de l'entreprise. II annonçait ainsi que les solidarités protectrices seraient inévitables dans un monde doté d'une puissance créative sans précédent (Hatchuel et Segrestin 2019).

D'autres auteurs s'inscrivent dans une perspective similaire. Frederick Taylor, qui au nom de la responsabilité de l'entreprise, s'oppose à l'organisation du travail comme un régime d'enchères marchandes auxquels les ouvriers seraient " librement » invités à répondre, en concurrence les uns avec les autres. En stabilisant l'activité ouvrière et sa rémunération, le Taylorisme contribua à l'idée d'un " contrat de travail » protecteur. Le modèle qu'il contestait est aujourd'hui massivement de retour avec les plateformes informationnelles de mobilité ou de livraison. Et pour lutter contre l'irresponsabilité de ce modèle, il faut reconstituer une bene gesta contemporaine en inventant un régime équivalent au contrat de travail, régime sans lequel les crises récurrentes du salaire aux pièces, si fréquentes dans le monde prétaylorien, reviendront sous des formes amplifiées.

Mary Parker Follett avait aussi montré que la bonne gestion présuppose des "relations circulaires » (O'connor 2011) car elles permettent une créativité collective tant sur la fixation des objectifs que sur celle des moyens. Elle étend ainsi la théorie administrative à des actions collectives fondées sur l'engagement volontaire et bénévole. Au sein de ces formes " associatives ", les principes Cicéroniens de la bene gesta res publicae (bonne gestion des choses publiques) retrouvent une nouvelle fraîcheur. En effet, les valeurs citoyennes et solidaires qui fondent ces actions humanitaires peuvent

\footnotetext{
${ }^{16}$ Comme, par exemple, ce projet de « métal qui ne se dilate pas » que le physicien Charles-Edouard Guillaume propose en 1896 à Fayol. II mènera à la découverte de l'Invar, une des plus belles réussites industrielles de Fayol et qui vaudra à Guillaume le prix Nobel de Physique en 1920.
} 
se lire à la fois comme principes d'efficacité et comme principes de responsabilité ! Parallèlement, Maurice Hauriou distinguait entre l'Etat comme puissance publique et l'Etat comme " administration de services publics ". Distinction qui le conduit à retrouver une théorie de la gestion administrative comme " espace de relations » entre Etat et citoyens ${ }^{17}$.

\section{Conclusion : science fondamentale et inventions civilisationnelles.}

Après la seconde guerre mondiale, le développement du "management " s'est construit sur un double oubli. Oubli, de l'histoire longue que les termes de gestion et d'administration portaient en héritage. Oubli aussi des inventions des théoriciens qui de 1895 à 1939 organisent la restauration des " sciences de gestion » (O'connor 2011) et l'invention de l'entreprise moderne.

Des années 60 à nos jours, une très large part des travaux en management se concentre sur l'explication statistique ou structurale de la performance des entreprises, cette dernière étant dogmatiquement définie ${ }^{18}$. Le développement d'une théorie unifiée des organisations était plus prometteur. Celle-ci aurait pu retrouver la logique universaliste et unificatrice de la bene gesta. Mais la spécialisation disciplinaire qui marque le monde académique à partir des années 80 , a limité le travail transdisciplinaire nécessaire. En outre la persistance de l'économicisme a favorisé l'étanchéité entre science du management, science des organisations, socio-économie des organisations et sciences juridiques. La critique que l'on peut adresser à cette évolution académique reste d'abord celle d'Urwick: l'absence d'un projet unificateur ${ }^{19}$ et leur faible fécondité théorique malgré une accumulation d'analyses empiriques. Mais la recherche en management a aussi, et surtout, été dans l'incapacité à résister doctrinalement à la financiarisation théorique et normative des entreprises qui débute dans les années 80 et aboutira à la crise de 2008.

Après la crise de 2008, une nouvelle définition de l'entreprise et de sa mission,

En deux décennies, la réduction de la performance des entreprises au seul rendement actionnarial a balayé les acquis théoriques et protecteurs du début du siècle. La notion même d'entreprise moderne a été remplacée par des doctrines de gouvernance (Corporate governance) qui ont érodé l'autorité des dirigeants à mesure que leur rémunération explosait et provoqué une détérioration intense des relations internes et externes dans les entreprises (Segrestin et Hatchuel 2012). Par ailleurs, cette financiarisation a lourdement pesé sur la capacité d'innovation des entreprises au moment où, avec l'accélération et l'intensification des révolutions techniques celle-ci devenait vitale pour leur survie, pour celle des Etats, et pour relever les grands défis environnementaux (Hatchuel et al. 2017).

Une des grandes retombées du programme de refondation de 2001 a été l'implication de chercheurs en gestion dans la redéfinition récente de "l'entreprise " à travers un droit des sociétés remanié et la création des sociétés à raison d'être et à mission ${ }^{20}$. II y a là une victoire posthume de l'unité

\footnotetext{
17 Rappelons aussi les thèses d'un Léon Bourgeois qui théorise la " solidarité " comme " bene gesta " des sociétés modernes parce qu'elle conditionne la participation de tous à l'efficacité collective et parce qu'elle exprime simultanément la responsabilité de tous vis-à-vis de chacun.

${ }^{18}$ C'est ainsi que la capacité d'innovation des entreprises restera un point aveugle du management jusqu'aux années 2000.

${ }^{19}$ Il existe depuis quelques années des tentatives intéressantes d'unification des sciences sociales vers une science sociale (Caillé et al. 2017), mais le projet d'une théorie de l'action collective n'y figure pas tant la séparation entre efficacité et responsabilité reste profondément installée.

${ }^{20}$ La société à mission est une qualité des sociétés commerciales qui a été créée par la Loi française en Mai 2019. Elle s'inspire notamment des travaux conduits au Collège des Bernardins (Hatchuel et Segrestin 2012) et
} 
scientifique que recherchait Fayol. En réinventant/responsabilisant l'entreprise par les engagements qu'elle prend, au-delà des actionnaires, vis-à-vis des sociétés et des milieux naturels où elle agit, on rapproche action publique et action privée dans une même conception de la bene gesta (Segrestin et Levillain 2018). Ce faisant on ne se prive ni de l'échange marchand, ni de la capacité entrepreneuriale. On entre plutôt dans un monde post-hegelien (Levillain et al. 2014), où les actions collectives " privées " prennent conscience de leur puissance d'agir dans le processus civilisationnel et des responsabilités qui en découlent. Le profit marchand n'est plus alors qu'une logique, parmi d'autres, de la construction de l'action collective. De plus son mode de calcul et de répartition découle comme l'enseignait Fayol, des nécessités de la prévoyance et de l'union du "corps social »de l'entreprise, ce dernier étant étendu aux parties prenantes concernées.

\section{Une axiomatique générative pour une science refondée}

Fort heureusement, le champ des sciences de gestion a connu aussi, à partir des années 80 , une prise de conscience épistémologique accrue (Martinet 88) et une capacité d'invention théorique réelle. Aujourd'hui, on peut lire ces courants de recherche comme autant de tentatives pour reconstruire des théories de la rationalité et de la responsabilité adaptée aux menaces contemporaines. Sans être exhaustif, on peut évoquer deux voies de recherche, que nous savons maintenant indissociables et dont les impacts ont été multiples (Hatchuel et al. 2017).

- d'une part, les explorations de nouvelles rationalités plus conceptives, plus sensibles et plus écologiques qui éclairent les conditions de la création collective,

- d'autre part, les explorations de nouveaux principes de responsabilité conduisant à de nouveaux collectifs ou de nouvelles organisations, plus ouvertes, plus participatives dans lesquelles les logiques d'appartenance, de solidarité et de reconnaissance citoyenne ou politique sont repensées pour répondre à de nouvelles exigences territoriales, biographiques ou éthiques. ${ }^{21}$

In fine, il est acquis que le projet des "sciences de gestion " va maintenant bien au-delà du champ du « management » (Martinet et Pesqueux 2013). Pour nous, il débouche, du point de vue théorique, sur un cadre axiomatique de portée transdisciplinaire et qui échappe au piège conceptuel dans lequel l'oubli de l'héritage antique et l'économicisme généralisé nous avaient plongés. Le postulat de la non-séparabilité entre rationalité et responsabilité se révèle à la fois émancipateur et génératif. II installe une double interdépendance entre des notions que la modernité libérale a dogmatiquement posées comme indépendantes. Cette avancée est particulièrement adaptée aux défis contemporains. Par exemple : qu'est-ce aujourd'hui qu'une production d'énergie " efficace » ? peut-on la définir sans poser le problème du changement climatique? A l'évidence non. Autre exemple: qu'est-ce aujourd'hui qu'une justice "responsable "? peut-on la définir sans évoquer l'efficacité des procédures, ne serait-ce que leur durée ? Enfin, la prise en compte de notre responsabilité vis à vis des règnes naturels (animaux, plantes, milieux, planète...) peut-elle se passer d'un principe de rationalité créatrice pour notre action sur la nature?

permet un gouvernement d'entreprise beaucoup plus engagé dans la réalisation d'objectifs sociaux et environnementaux. La théorie de la mission comme principe d'efficacité et comme principe de responsabilité est cohérente avec les principes de la bene gesta. Elle actualise aussi cette dernière pour répondre aux défis contemporains (Segrestin et Levillain 2019).

${ }^{21}$ A l'occasion de multiples colloques, le mouvement de la Prospective du présent, autour d'Edith Heurgon, a donné une forte impulsion à ces nouvelles logiques collectives. 
Comme pour toute axiomatique, cette formulation a de nombreux équivalents ${ }^{22}$ et ses implications sont multiples. Le postulat de non-séparabilité s'adresse à toute forme d'agir collectif, y compris à tout projet de connaissance collective, car celui-ci n'est qu'une forme particulière d'agir collectif ! On peut donc attester qu'un monde académique qui ne reposerait que sur un principe de productivité scientifique n'est pas viable. On peut aussi s'appuyer sur l'axiome de non-séparabilité pour rejeter l'économicisme et repenser la constitution des sciences sociales. Ou pour interroger les pensées contemporaines de la vulnérabilité (Care) ou du don (Caillié 2018) en remarquant qu'elles gagneraient à clarifier la "bene gesta " associée, c'est-à-dire le couplage entre efficacité et responsabilité qu'elles se proposent d'explorer.

En définitive, un projet de recherche en sciences de gestion, y compris dans son volet d'analyse empirique, ne peut plus se définir comme la recherche de la meilleure efficacité ou comme la recherche de la responsabilité la plus adaptée. II devrait en toute rigueur s'énoncer avec deux formulations récursives reliant les moyens et les fins : quelles formes d'efficacité et de responsabilité sont cohérentes avec le futur collectivement désiré (science des moyens) ? Quelles formes de responsabilité et d'efficacité permettent d'élaborer collectivement un futur désiré (science des fins) ?

Après l'épuisement contemporain des grandes idéologies modernes et la prise de conscience du piège de l'économicisme, une théorie fondamentale et unifiée de l'action collective devient indispensable. Elle seule offre un cadre adapté pour qualifier les dérives collectives et inventer solidairement des réponses efficaces/responsables face aux risques vitaux qui menacent la vie sur la planète. Car, réconcilier justice sociale et transition écologique, c'est poser, deux mille ans après Cicéron, la question d'une nouvelle bene gesta res publicae, à ceci près que la vie sur la planète est devenue notre res publicae (chose publique) la plus précieuse.

\section{Références}

Bertilorenzi, M., Dubruc, N., Passaqui, J.P., Henri Fayol. Les multiples facettes d'un manager, Presses des Mines 2019.

Caillé, A., Chanial, P., Dufoix, S., Vanderbergue, F., (2017), Des sciences sociales à la science sociale globalisée, Colloque de Cerisy. Editions Le Bord de l'eau.

Crété, M.. (2018), "La " gestion » à l'époque romaine : naissance d'une nouvelle catégorie de l'action collective ", Entreprises et histoire, vol. 90, no. 1, pp. 164-177.

David, A., Hatchuel A., Laufer R. (2002), Les nouvelles fondations des sciences de gestion, Vuibert Fnege Paris.

Fayol, H. (1917). Administration Industrielle et Générale Paris, Dunod et Pinat.

Fayol, H., (1949), General and Industrial Management. London: Sir Isaac Pitman and Sons. Traduction de Constance Storrs. Préface de Lyndall Urwick.

Hatchuel, A. (2001a). Quel horizon pour les sciences de gestion ? Vers une théorie de l'action collective. In A. David, A. Hatchuel, \& R. Laufer (Eds.), Les nouvelles fondations des sciences de gestion, éléments d'épistémologie en management. Paris: Vuibert.

\footnotetext{
${ }^{22}$ L'axiomatique rationalité/responsabilité est formellement équivalente à l'axiomatique savoirs/relations que nous avons beaucoup étudiée ailleurs, et qui donne une formulation plus abstraite (Hatchuel 2001, 2005)
} 
Hatchuel, A. (2001b). Towards Design Theory and expandable rationality : the unfinished program of Herbert Simon. Journal of Management and Governance, Vol. 5(n³-4), pp. 260273.

Hatchuel, A. (2005). Towards an epistemology of collective action: management research as a responsive and actionable discipline. European Management Review, 2, pp. 36-47.

Hatchuel A., Weil B., (2014), Les nouveaux régimes de la conception. Langages, théories, métiers, Colloque de Cerisy, Hermann 2è édition Vuibert Paris.

Hatchuel, A., Le Masson, P., Segrestin B., Weil, B. (2017), Comprendre et soutenir l'innovation contemporaine: Théorie de la conception et métabolisme des nouveaux collectifs. La lettre de I'InSHS, CNRS, Mai 2017.

Hatchuel A., Weil, (2018), La " gestion » à l'époque romaine, Entreprises et histoire, vol. 90, no. 1, 2018, pp. 161-163. Avant-propos à l'article : Crété, Moïra. "La " gestion » à l'époque romaine : naissance d'une nouvelle catégorie de l'action collective », Entreprises et histoire, vol. 90 , no. 1,2018 , pp. 164-177.

Hatchuel A., Segrestin B. (2019), A century old and still visionary : Fayol's innovative theory of management, European Management Review, Vol. 16, 2, 399-412.

Le Masson, P., Hatchuel, A., \& Weil, B. (2010). Strategic Management of Innovation and Design: Cambridge University Press.

Levillain, K., Segrestin, B., Hatchuel A., (2014), Repenser les finalités de l'entreprise. La contribution des sciences de gestion dans un monde post-hégélien, Revue Français de Gestion n²45, Novembre-décembre 2014.

Martinet A.C., 1990, (ss. la dir), Epistémologie et Sciences de Gestion, Economica Paris

Martinet A.C., Y. Pesqueux, (2013), Epistemologie des sciences de gestion, FNEGE, Vuibert.

O'Connor S. E., (2011), Creating new Knowledge in Management. Appropriating the field's lost foundations, Stanford University Press.

Emmanuel Saint-Fuscien, $A$ vos ordres ? La relation d'autorité dans l'armée française de la Grande Guerre, Paris, EHESS, coll. « En temps \& lieux », 2011, 311 p.

Segrestin, B., \& Hatchuel, A. (2012), Refonder l'entreprise, Paris: Le Seuil.

Segrestin, B. , Levillain K., (ss. la dir.) (2019), La mission de l'entreprise responsable, Presses des Mines.

Urwick L., (1949), Préface à l'ouvrage : Fayol, H., (1949), General and Industrial Management. Traduction de Constance Storrs. London: Sir Isaac Pitman and Sons. 
